\title{
Nuclear medicine innovations help (drive) healthcare (benefits)
}

\author{
Marcus Hacker - Thomas Beyer • Richard P. Baum • Antonis Kalemis • \\ Adriaan A. Lammertsma • Val Lewington • Jean-Noël Talbot • \\ Fred Verzijlbergen
}

Published online: 22 November 2014

(C) Springer-Verlag Berlin Heidelberg 2014

Nuclear medicine is an integral part of modern healthcare. It uses biomolecules tagged with radioactive isotopes that can recognize different molecular targets in the body or seek out hallmarks of malignant and benign conditions. The distribution of trace amounts of a radioactively labelled molecule, known as a radiotracer, can be mapped and followed noninvasively anywhere in the human body using a dedicated system, such as a single photon emission computed tomography (SPECT) system or a positron emission tomography (PET) system. This trace approach provides longitudinal sets of volumetric and quantitative images that can be used to diagnose a wide range of diseases and/or assess response to disease-specific treatments. Replacing these diagnostic radionuclides with alternative nuclides that emit a different type of radiation converts imaging tracers into drugs that deliver potent and targeted molecular treatment. Recent advances

\section{Hacker}

Division of Radiology and Nuclear Medicine,

Department of Biomedical Imaging and Image-guided Therapy,

Medical University of Vienna, Vienna, Austria

\section{T. Beyer $(\square)$}

Center for Medical Physics and Biomedical Engineering,

General Hospital Vienna, Medical University of Vienna,

Waehringer Guertel 18-20/4 L, 1090 Vienna, Austria

e-mail: thomas.beyer@meduniwien.ac.at

\section{R. P. Baum}

THERANOSTICS Center for Molecular Radiotherapy and Molecular Imaging (PET/CT), ENETS Center of Excellence, Zentralklinik Bad Berka, Germany

\section{A. Kalemis}

Advanced Molecular Imaging, Philips Healthcare, Guildford GU2

8XH, Surrey, UK

\section{A. Kalemis}

European Industrial Association for Nuclear Medicine and Molecular Healthcare (AIPES eeig), Brussels, Belgium have extended the range of molecular radiotherapies, which are now applied across a broad spectrum of diseases from arthritis and benign thyroid diseases to many types of cancer. As such, nuclear medicine is in a key position to bring personalized or image-guided therapy into routine clinical practice.

This key position is supported by the rapidly expanding fields of radiochemistry and radiopharmacy with novel kitlike or cassette-type labelling techniques which allow shorter turn-around times from the identification of therapeutic targets to the design and evaluation of associated radiotracers, resulting in lower development costs. Over the past 5 years, significant technological breakthroughs in imaging hardware and image processing algorithms have also been introduced, enabling specific radiotracer imaging for improved characterization of targeted cellular and functional processes in clinical

\section{A. A. Lammertsma}

Department of Radiology and Nuclear Medicine,

VU University Medical Center, Amsterdam, Netherlands

\author{
V. Lewington \\ Clinical Therapeutic Nuclear Medicine at King's College London, \\ London, UK
}

J.-N. Talbot

Department of Nuclear Medicine, University Pierre and Marie Curie, Paris, France

F. Verzijlbergen

Department of Nuclear Medicine Rotterdam, Erasmus Medical Center, Rotterdam, Netherlands 
routine. Recent advances in PET detector design have resulted in tomographs with improved lesion detection capabilities through better contrast and spatial resolution performance, that can also be attributed to the incorporation of stable timeof-flight (ToF) performance and larger axial fields-of-view.

In parallel with these hardware developments, advanced implementations of updated image reconstruction algorithms have further improved image quality and, just as importantly, quantitative performance. Associated developments include spatial resolution modelling, incorporation of prior object information in the reconstruction algorithms, better motion estimation and correction, and improved scatter correction using simulations and anatomical priors. When combined with highly specific radiopharmaceuticals with elevated target-to-background ratios, these technological advances will significantly increase detectability of very small lesions and visualization of small cerebral structures. Furthermore, increased system sensitivity will enable higher patient throughput or the application of more sophisticated PET protocols, such as dynamic PET or multitracer scans, keeping clinical throughput the same.

Likewise, the range of applications of SPECT imaging has benefited from the adoption of solid-state detectors (e.g. $\mathrm{CdZnTe}$ ) that provide better energy resolution and, if incorporated into organ-specific SPECT systems, higher volume sensitivity than scintillator-based detector systems. For applications in nuclear cardiology the higher system sensitivity translates into very short acquisition times of around $2 \mathrm{~min}$ or, if acquisition time remains unchanged, in significantly reduced patient exposure of less than $1 \mathrm{mSv}$ for a myocardial SPECT examination.

Next to technical developments and impressive advances in diagnostic quality, nuclear medicine also adds to the armamentarium of therapeutic options through the concept of theranostics, a combination of therapy and diagnostics using the same biomolecule labelled with different radioisotopes to provide inherently synergistic imaging and therapy. Next to treating thyroid cancer, the validity of theranostics in modern healthcare has been demonstrated in neuroendocrine tumours (NET). Here, somatostatin analogues are used as ligands and ${ }^{68} \mathrm{Ga} /{ }^{90} \mathrm{Y}$ or ${ }^{68} \mathrm{Ga} /{ }^{177} \mathrm{Lu}$ as pairs of diagnostic/therapeutic radionuclides. In patients with progressive NET, fractionated, personalized peptide radioreceptor therapy (PRRT) with lower doses of radioactivity given over an extended period of time results in excellent therapeutic responses. At the same time, severe haematological and renal toxicities are reduced resulting in a significant improvement in quality of life. PRRT has also proven to be highly effective in the management of advanced NET. These facts attest to the power of personalized medicine. This concept is further strengthened by recognizing the importance of visualizing molecular therapeutic targets, such as the expression of hormone receptors in breast cancer, for improved patient and therapy selection.
Furthermore, recent progress in genomic exploration of cancers as well as in detection of circulating cancer cells or abnormal DNA and mRNA has been demonstrated, thus, rendering in vivo nuclear imaging as a core technique for whole-body lesion detection and tissue characterization.

In summary, nuclear medicine provides non-invasive tools to monitor and predict responses to therapy. As such, it gives an opportunity to select the best therapy for the individual patient. Ultimately, this will lead to improved quality of life and, at the same time, a reduction in healthcare costs. While this is not new to members of the nuclear medicine community, it must be recognized that nuclear medicine is still seen as something obscure or even dangerous. Therefore, there is a need to align internally and champion the following key messages:

1. Molecular imaging is effective and safe in diagnosis, staging, prognostication and follow-up of many clinical indications

This has been demonstrated in prospective trials of $\left[{ }^{18} \mathrm{~F}\right] \mathrm{FDG}$ PET in lung cancer (diagnosis, staging and outcome prediction) and lymphoma patients (therapy response). These studies also demonstrated a clear diagnostic benefit over anatomical imaging (CT, MRI) alone. Through the application of only trace amounts of radiolabelled biomolecules at picomolar concentrations, PET imaging does not affect patient safety, as has been known since the early 1980s. Likewise, large-scale nuclear medicine therapy studies with $\beta$-emitters including ${ }^{131} \mathrm{I}$ and ${ }^{177} \mathrm{Lu}$ have shown only minor levels of side effects if any.

2. Molecular imaging can avoid unnecessary treatments and reduces patient discomfort and side effects

Nuclear medicine molecular imaging methods enable the selection of patients for targeted therapies by proving the presence or absence of therapeutic targets in vivo. For example, several $\left[{ }^{18} \mathrm{~F}\right]$ fluoroestradiol imaging studies of the oestrogen receptor status have consistently shown high negative predictive value: in the absence of significant tracer accumulation, the probability of response to endocrine therapy is very low and alternative therapy approaches should be considered, thereby avoiding unnecessary drug treatment and its associated side effects. Similar observations have also been reported for $\left[{ }^{89} \mathrm{Zr}\right]$ trastuzumab and receptor-specific tracers, which however still lack systematic investigation using structured prospective trials.

3. Molecular imaging helps individualize patient treatment

Through growing evidence from genetic profiling it is understood that cancer is not a single homogeneous disease, but that it is represented by a multitude of genetic alterations and changes at the cellular level. Adenocarcinomas of the lung, for example, can contain several different gene 
mutations such as BRAF, EGFR, KRAS or others, all of which require different treatment strategies.

Today the identification of potential therapeutic targets requires biopsies of randomly assigned tumour regions, whole tumours and multiple tumour lesions. Modern nuclear medicine techniques enable serial and non-invasive visualization of functional pathologies at a molecular level. Therefore, imaging modalities such as PET and SPECT are the most appropriate techniques for patient-centred tissue characterization prior to and following therapy.

4. Molecular imaging can help reduce healthcare costs

Pioneering work on cost effectiveness of nuclear medicine studies has been performed in the field of nuclear cardiology, where it was clearly shown that a clinical approach including myocardial scintigraphy as a gatekeeper for invasive coronary angiography or revascularization results in significant cost savings while offering patients a clear outcome benefit. There is similar evidence for the diagnosis and treatment of patients lung cancer.

5. Theranostics is a concept unique to nuclear medicine

Molecular imaging and therapy (also known as therapy and diagnostics: theranostics) using the same biomolecule labelled with two different radioisotopes is a concept unique to nuclear medicine. Examples include radiopeptides such as the somatostatin analogues and PSMA, monoclonal antibodies against CD20, the combination of bone scintigraphy and ${ }^{223} \mathrm{Ra}$ therapy of bone metastases, and radionuclide imaging and therapies using ${ }^{123}$ I and ${ }^{131} \mathrm{I}$, respectively.

Nuclear medicine techniques are vital for the development of new drugs and to accelerate research into future treatments of disorders including schizophrenia, movement disorders, Alzheimer's disease, coronary artery disease and inflammatory/infectious disease. Associated registration studies entail multicentre effort and therefore require support from independent clinical bodies, such as the EORTC, whilst financial backing from international organizations, industry and, ideally, health insurance companies is also needed.

Molecular imaging is effective and safe for the diagnosis, staging, prognostication and follow-up of many clinical indications. Molecular imaging is a medical imaging modality that helps distinguish early responders from nonresponders, thus, saving patients from unnecessary treatments, which in turn reduces patient discomfort and side effects. This may be the most effective way to personalize therapy to cut down on the waste of financial healthcare resources associated with the prescription of expensive therapies for patients without knowing whether they have the appropriate disease genotype that many newer therapies are targeting. "What you see is what you treat" should be the overarching objective of our efforts. For sure, this is a very facile statement whilst being easy to comprehend by politicians and health insurance companies.

Irrespective of the perceived and known strengths of our field, the nuclear medicine community should align and together with oncologists, haematologists, endocrinologists and other therapy specialists advocate a policy change in order to lessen the stringent requirements for clinical adoption of new radiopharmaceuticals. There is an urgent need to increase the awareness of the full scope of nuclear methodology. This requires active communication and co-operation with other medical disciplines and associations, much like participation in lobbying efforts. The failing clinical acceptance of $\left[{ }^{90} \mathrm{Y}\right]$ Zevalin (anti-CD20) therapy, for example, has conclusively shown that co-operation with the referring physicians is at least as important as proof-of-concept evidence in high quality trials. Therefore, active involvement and improved communication among referring physicians, nuclear medicine specialists and industrial partners is essential.

Quantitative and molecular nuclear medicine imaging should be an integral part of the design of intelligent, costeffective clinical trials at the cutting edge of research. It does not need an evangelist to relay this message, but each of us should act as a champion of an exciting and rewarding medical discipline.

Acknowledgments This editorial is the essence of an international symposium and roundtable discussions organized by AIPES on 9 May 2014 in Brussels.

Conflicts of interest All authors received support for travel expenses from AIPES. 